\title{
Genome analysis reveals three genomospecies in Mycobacterium abscessus
}

Mohamed Sassi ${ }^{1}$ and Michel Drancourt ${ }^{1,2^{*}}$

\begin{abstract}
Background: Mycobacterium abscessus complex, the third most frequent mycobacterial complex responsible for community- and health care-associated infections in developed countries, comprises of $M$. abscessus subsp. abscessus and M. abscessus subsp. bolletii reviously referred as Mycobacterium bolletii and Mycobacterium massiliense. The diversity of this group of opportunistic pathogens is poorly described.

Results: In-depth analysis of 14 published M. abscessus complex genomes found a pan-genome of 6,153 proteins and core-genome of 3,947 (64.1\%) proteins, indicating a non-conservative genome. Analysing the average percentage of amino-acid sequence identity (from 94.19\% to 98.58\%) discriminates three main clusters C1, C2 and C3: C1 comprises strains belonging to $M$. abscessus, C2 comprises strains belonging to $M$. massiliense and C3 comprises strains belonging to M. bolletii; and two sub-clusters in clusters $\mathrm{C} 2$ and C3. The phylogenomic network confirms these three clusters. The genome length (from 4.8 to 5.51-Mb) varies from 5.07-Mb in C1, 4.89-Mb in C2A, 5.01-Mb in C2B and 5.28-Mb in C3. The mean number of prophage regions (from 0 to 7 ) is 2 in $C 1 ; 1.33$ in $C 2 A ; 3.5$ in $C 2 B$ and five in C3. A total of 36 genes are uniquely present in C1, 15 in C2 and 15 in C3. These genes could be used for the detection and identification of organisms in each cluster. Further, the mean number of host-interaction factors (including PE, PPE, LpqH, MCE, Yrbe and type VII secretion system ESX3 and ESX4) varies from 70 in cluster C1, 80 in cluster C2A, 74 in cluster C2B and 93 in clusters C3A and C3B. No significant differences in antibiotic resistance genes were observed between clusters, in contrast to previously reported in-vitro patterns of drug resistance. They encode both penicillin-binding proteins targeted by $\beta$-lactam antibiotics and an Ambler class A $\beta$-lactamase for which inhibitors exist.
\end{abstract}

Conclusions: Our comparative analysis indicates that M. abscessus complex comprises three genomospecies, corresponding to $M$. abscessus, $M$. bolletii, and $M$. massiliense. The genomics data here reported indicate differences in virulence of medical interest; and suggest targets for the refined detection and identification of M. abscessus.

Keywords: Mycobacterium abscessus, Mycobacterium bolletii, Mycobacterium massiliense, Araucaria, Mycobacteriophage, Prophage

\section{Background}

The non-tuberculous mycobacterium Mycobacterium abscessus was long confused with Mycobacterium chelonae [1]. Other closely related species include Mycobacterium salmoniphilum [2], Mycobacterium immunogenum [3], Mycobacterium massiliense [4], Mycobacterium bolletii [5] and Mycobacterium franklinii [6] altogether forming the Mycobacterium chelonae-abscessus complex. This complex is the third most frequent mycobacterial complex

\footnotetext{
* Correspondence: michel.drancourt@univ-amu.fr

'Aix Marseille Université, URMITE, UMR63, CNRS 7278, IRD 198, Inserm 1095, Marseille, France

${ }^{2} U$ nité de Recherche sur les Maladies Infectieuses et Tropicales Emergentes, Faculté de Médecine, 27, Boulevard Jean Moulin, Marseille cedex 5, France
}

infecting humans in developed countries besides the Mycobacterium tuberculosis and Mycobacterium avium complexes [7,8]. Bibliometrics retrieving over 1,700 publications in the Medline database illustrates the fact that this complex is emerging, causing both sporadic cases and outbreaks of community-acquired and health-care associated infections [9]. Not only humans but also cats [10,11] and dolphins [12-14] are infected while fishes are uniquely infected by $M$. salmoniphilum $[2,15]$.

Current nomenclature is that the species $M$. abscessus comprises two subspecies named $M$. abscessus subsp. abscessus and M. abscessus subsp. bolletii. Later taxon accommodates isolates previously referred as $M$. bolletii or M. massiliense [16]. This nomenclature however may 
obscure the true diversity of mycobacteria in this complex. While the 16S rRNA gene yields an identical sequence for M. abscessus and M. bolletii, it shares $99 \%$ sequence identity with $M$. massiliense. RpoB gene sequencing founded the description of recent species [17-19] but yielded further conflicting results [20-22]. Multilocus sequencing analysis [23] and multispacer sequence typing [24] differentiated M. massiliense from $M$. bolletii. In this report, the previous nomenclature $M$. abscessus, $M$. bolletii and $M$. massiliense forming the $M$. abscessus complex, has been retained for clarity.

The availability of $39 M$. abscessus, $13 \mathrm{M}$. massiliense and two M. bolletii genomes in the National Center for BioInformatics (NCBI) genome database provides new opportunities to assess the diversity of this species. Here, we review 14 complete published $M$. abscessus complex genomes and compare them with the re-annotated M. tuberculosis $\mathrm{H} 37 \mathrm{Rv}$ genome (Table 1) in order to in-depth analyse the diversity of M. abscessus.

\section{Results and discussion}

M. abscessus complex pan- and core-genome

$M$. abscessus complex genomes comprise one circular chromosome. In addition, M. abscessus ATCC 19977 contains one 23-kb plasmid identical to the Mycobacterium marinum pMM23 plasmid, encoding mer operon and mercury reductase protein, which may confer resistance to organo-mercury compounds [25]. In order to normalize the predicted proteins and to minimize the differences of presence/absence of genes and length, coding sequences were predicted using prodigal software [26]. We identified a total of 70,309 protein-coding sequences which number varies from 4,651 to 5,079 in each genome (Table 2). The core-genome contains 57,172 protein sequences accounting for $64.15 \%$ of the pan-genome. This figure indicates a non-conservative genome contrary to that of Mycobacterium tuberculosis, a conservative-genome pathogen which core-genome accounts for $96.1 \%$ of the pan-genome [27]. Using orthoMCL [28] with a conservative parameter value of $50 \%$ sequence identity, we categorized these 70,309 proteins into 6,153 orthologous protein groups, including 3,947 core-genome groups and 55 strain-specific groups.

\section{M. abscessus complex diversity}

The average percentage of amino-acid sequence identity (AAI) of core proteins was determined as previously described [29]. The AAI values indicate that M. abscessus complex forms three main clusters: cluster 1 (C1) includes M. abscessus type strain and strains M93, 94, M152 and Go06; cluster 2 (C2) contains two subclusters: cluster $2 \mathrm{~A}$

Table 1 List of Mycobacterium abscessus genomes here studied

\begin{tabular}{|c|c|c|c|c|}
\hline Clusters & Organism & Isolated from & Geography & BioProject \\
\hline $\mathrm{C1}$ & M. abscessus Type strain & human knee infection & United States & PRJNA61613, PRJNA15691 \\
\hline C1 & M. abscessus M93 & $\begin{array}{l}\text { sputum sample from a Malaysian patient presenting } \\
\text { with a prolonged productive cough suggestive of } \\
\text { a bacterial lower respiratory tract infection }\end{array}$ & Malysia & PRJNA180393, PRJNA84203 \\
\hline C1 & M. abscessus M94 & $\begin{array}{l}\text { sputum sample of a Malaysian patient with } \\
\text { a persistent cough and fever and } \\
\text { consolidation in the chest radiograph }\end{array}$ & Malysia & PRJNA180394, PRJNA88149 \\
\hline C1 & M. abscessus M152 & acid-fast bacillus positive sputum of a Malaysian man & Malysia & PRJNA159789 \\
\hline C1 & M. massiliense strain GO 06 & undergone knee joint surgery & Brazil & PRJNA170732, PRJNA168263 \\
\hline $\mathrm{C} 2 \mathrm{~A}$ & M. massiliense Type strain & sputum specimen from hemoptoic pneumonia & Marseille & PRJNA180742, PRJNA65215 \\
\hline $\mathrm{C} 2 \mathrm{~A}$ & M. massiliense M18 & $\begin{array}{l}\text { lymph node biopsy specimen from a Malaysian } \\
\text { patient suspected of having tuberculous } \\
\text { cervical lymphadenitis }\end{array}$ & Malysia & PRJNA89593 \\
\hline $\mathrm{C} 2 \mathrm{~A}$ & M. massiliense M154 & $\begin{array}{l}\text { bronchoalveolar lavage fluid of a Malaysian } \\
\text { patient presenting with lower respiratory } \\
\text { tract infection }\end{array}$ & Malysia & PRJNA89603 \\
\hline C2B & M. abscessus $47 \mathrm{~J} 26$ & sputum sample from a patient with Cystis fibrosis & England & PRJNA179981, PRJNA73255 \\
\hline $\mathrm{C} 2 \mathrm{~B}$ & M. abscessus M115 & $\begin{array}{l}\text { sputum from a Malaysian patient presenting with } \\
\text { persistent cough and loss of body weight } \\
\text { suggestive of pulmonary tuberculosis }\end{array}$ & Malysia & PRJNA89601 \\
\hline $\mathrm{C} 2 \mathrm{~B}$ & M. abscessus M139 & $\begin{array}{l}\text { sputum sample of a } 26 \text {-year-old Nepalese } \\
\text { male presenting with hemoptysis }\end{array}$ & Nepal & PRJNA159701 \\
\hline $\mathrm{C} 2 \mathrm{~B}$ & M. abscessus M172 & putum isolate from a Malaysian patient & Malysia & PRJNA89599 \\
\hline C3A & M. bolletii Type strain & $\begin{array}{l}\text { respiratory tract specimen collected } \\
\text { in woman with hemoptoic pneumonia }\end{array}$ & Marseille & PRJNA180015, PRJNA73695 \\
\hline C3B & M. abscessus M24 & the bronchoalveolar lavage fluid of a Malaysian patient & Malysia & PRJNA89595 \\
\hline
\end{tabular}


Table 2 Mycobacterium abscessus core genome and unique genes

\begin{tabular}{lllll}
\hline Clusters & Organism & CDS & $\begin{array}{l}\text { Unique core } \\
\text { genome }\end{array}$ & $\begin{array}{l}\text { Total } \\
\text { genes }\end{array}$ \\
\hline \multirow{2}{*}{ C1 } & M. abscessus & - & 36 & $\mathbf{1 8 0}$ \\
& M. abscessus T & 4954 & - & - \\
& strain GO 06 & 4944 & - & - \\
& M93 & 4733 & 11 & 11 \\
& M94 & 4841 & 10 & 10 \\
& M152 & 4762 & - & - \\
& M. massiliense & - & $\mathbf{1 5}$ & $\mathbf{1 0 7}$ \\
C2A & M. massiliense T & 4962 & 3 & 3 \\
& M18 & 4663 & 8 & 8 \\
& M154 & 4651 & - & - \\
C2B & 47 J26 & 4766 & - & - \\
& M115 & 4802 & 4 & 4 \\
& M139 & 4754 & 4 & 4 \\
& M172 & 5079 & 20 & 20 \\
& M. bolletii & - & $\mathbf{1 5}$ & $\mathbf{3 0}$ \\
C3A & M. bolletii T & 4733 & 9 & 9 \\
& M24 & 4960 & 23 & 23 \\
& M. abscessus core genome & - & $\mathbf{3 , 9 4 7}$ & $\mathbf{5 7 , 1 7 2}$ \\
\hline & & & & \\
& & &
\end{tabular}

(C2A) includes $M$. massiliense type strain and strains M154 and M18; cluster 2B (C2B) includes strains 47 J26, M115, M172 and M139; cluster 3 (C3) includes two subclusters: cluster 3A (C3A) includes $M$. bolletii type strain and cluster 3B (C3B) includes M. bolletii strain M24 (Table 3).
M. abscessus complex proteomes were further aligned using Mauve software [30] to infer phylogeny using the Neighbor-Net algorithm in the package SplitsTree4 [31]. The phylogenomic network confirms the three clusters C1, C2 and C3 (Figure 1A). A phylogenomic tree based on gene content (i.e., the presence or absence of orthologs) (Figure 1B) organizes $M$. abscessus differently from the whole genome concatenated tree (Figure 1A) or even the phylogenetic tree based on rpoB gene sequence (Figure 1C). Phylogenomic analysis indicates that the $M$. abscessus gene repertoires have different evolutionary histories and suggests that differential gene loss and lateral gene acquisition are playing important roles in the evolution of some $M$. abscessus strains. Notably, the situation of strain Go06 is confusing, as it presents $98.4 \%$ AAI with $M$. abscessus type strain in $\mathrm{C} 1$ (Figure $1 \mathrm{~A}$ ) whereas its rpoB gene sequence and single nucleotide polymorphisms analysis are indicative of $M$. massiliense [8,32]. Our analyses indicate that strain Go06 have an ambiguous classification as a chimera between $M$. abscessus and $M$. massiliense and is the only example compatible with a lateral transfer of $r p o \mathrm{~B}$ gene.

Functional analysis using Clusters of Orthologous Groups database (COG) [33] and BLASTP indicates unique genes in $\mathrm{C} 1$, encoding hypothetical proteins, proteins implicated in transcription, energy production and transport, carbohydrate metabolism and transport, lipid metabolism, nucleotide metabolism and transport, amino-acid metabolism and transport, post-translational modification and inorganic ion transport and metabolism (Table 2, Figure 2). Within C2, unique genes encode hypothetical proteins, proteins implicated in transcription and lipid metabolism. Within C3,

Table 3 Average nucleodite identity and characteristics of Mycobacterium abscessus genomes

\begin{tabular}{|c|c|c|c|c|c|c|c|}
\hline Clusters & & Strains & $\begin{array}{l}\text { Genome } \\
\text { lenght } \mathrm{Mb}\end{array}$ & $\begin{array}{l}\text { Genome } \\
\text { GC\% }\end{array}$ & $\begin{array}{l}\text { AAI Vs } \\
\text { M. abscessus T }\end{array}$ & $\begin{array}{l}\text { AAI Vs } \\
\text { M. bolletii T }\end{array}$ & $\begin{array}{l}\text { AAI Vs } \\
\text { massiliense T }\end{array}$ \\
\hline \multirow[t]{5}{*}{ M. abscessus } & $\mathrm{C} 1$ & M. abscessus $T$ & 5,09 & 62,7 & 100,00 & 95,56 & 94,74 \\
\hline & & M93 & 5,08 & 64,2 & 97,30 & 95,35 & 94,76 \\
\hline & & M94 & 5,1 & 64,2 & 97,56 & 95,67 & 94,79 \\
\hline & & M152 & 4,91 & 64,1 & 98,59 & 96,33 & 95,73 \\
\hline & & strain GO 06 & 5,07 & 64,2 & 98,35 & 95,23 & 95,64 \\
\hline \multirow[t]{7}{*}{ M. massiliense } & $\mathrm{C} 2 \mathrm{~A}$ & M. massiliense $T$ & 5,2 & 64,2 & 95,56 & 96,13 & 100,00 \\
\hline & & M18 & 4,89 & 64,2 & 96,66 & 96,09 & 97,57 \\
\hline & & M154 & 4,8 & 64,1 & 96,14 & 95,81 & 97,26 \\
\hline & $\mathrm{C} 2 \mathrm{~B}$ & M115 & 4,98 & 64,1 & 96,16 & 95,36 & 96,92 \\
\hline & & M172 & 5,2 & 64,2 & 95,30 & 94,93 & 96,17 \\
\hline & & M47 J26 & 4,87 & 64,1 & 96,23 & 95,74 & 96,93 \\
\hline & & M139 & 5,05 & 64,1 & 95,94 & 95,64 & 96,88 \\
\hline \multirow[t]{2}{*}{ M. bolletii } & C3A & M. bolletii $T$ & 5,05 & 64,2 & 94,51 & 100,00 & 95,33 \\
\hline & C3B & M24 & 5,51 & 64,2 & 94,91 & 96,47 & 94,20 \\
\hline
\end{tabular}




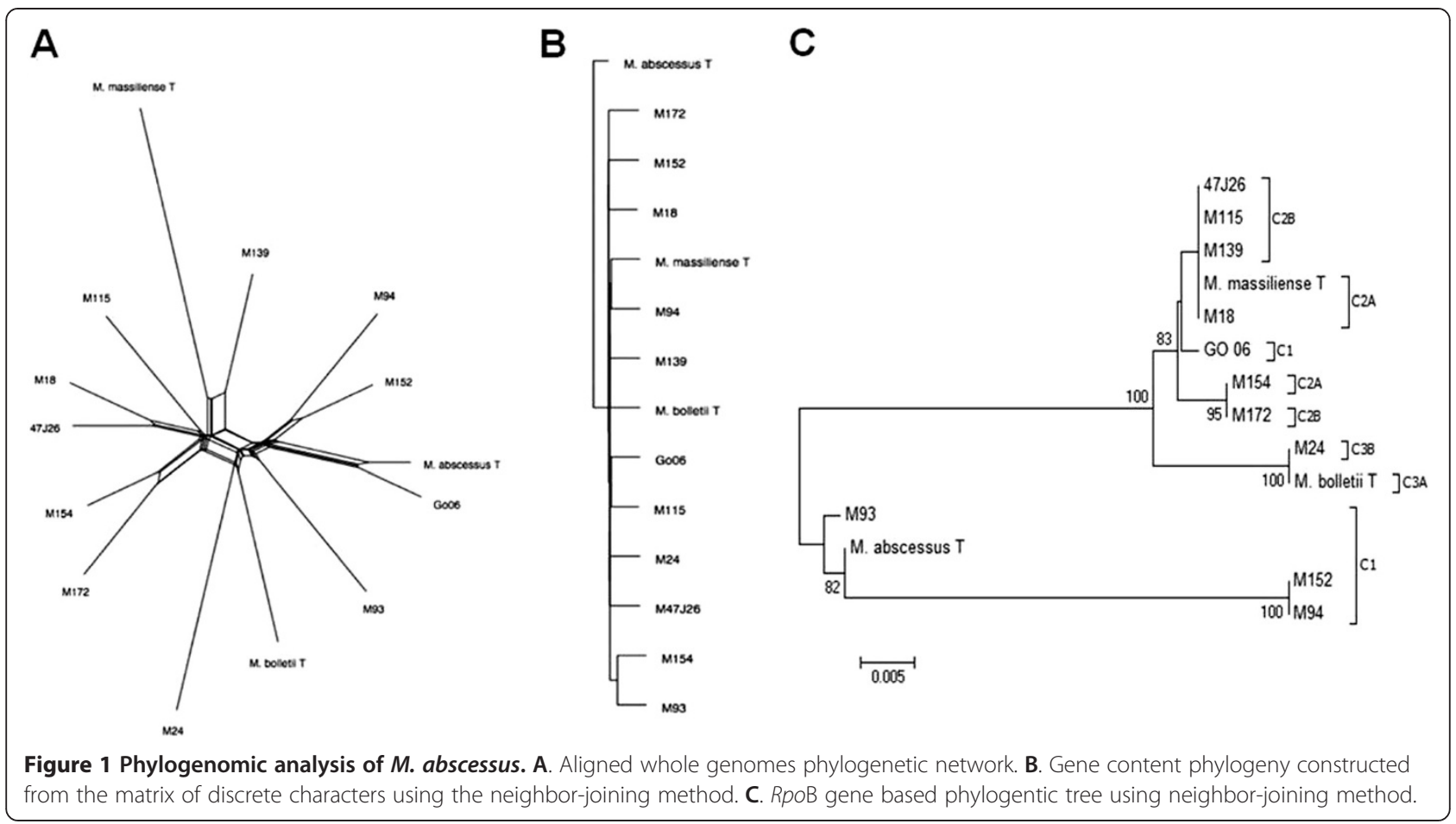

unique genes encode hypothetical proteins, proteins implicated in amino-acid metabolism and transport and translation. These unique genes could base a refined identification of the three genomospecies. However, we could not exclude that these unique genes could be due to a coding sequence, which arose de novo, to HGT or gene loss for the other subspecies. In the case of absence of a gene, this could also be due to a real loss or to an assembly artefact.

C1 strains have been isolated from American and Malaysian patients suffering knee infection and lower

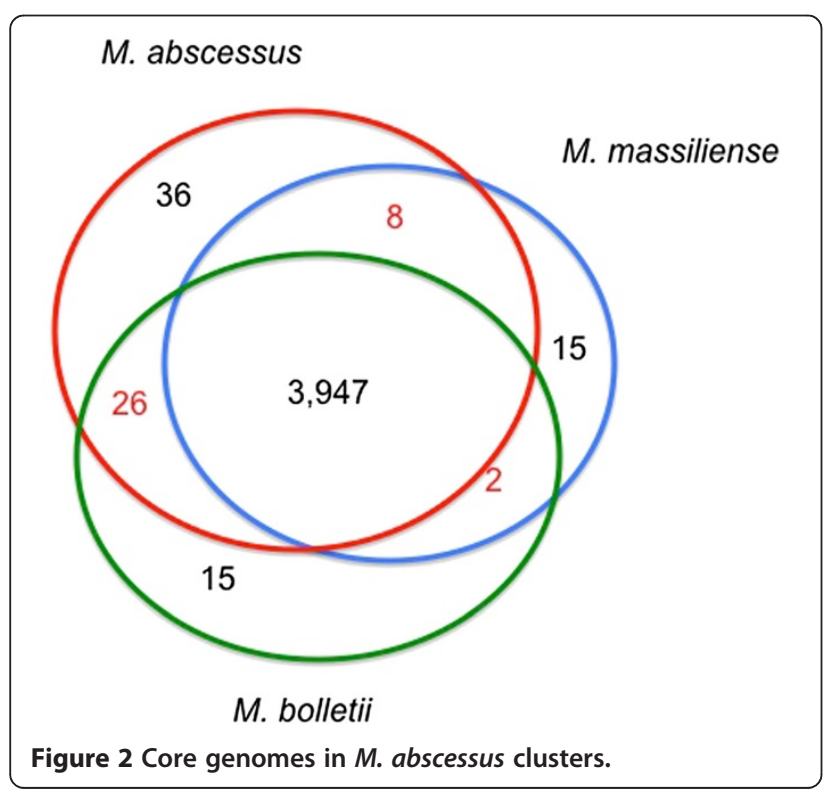

respiratory infection, respectively (Table 1). C2A strains were isolated from Malaysian and French patients suffering severe, respiratory tract infections. C2B strains were isolated from Nepalese, Malaysian and English patients suffering respiratory tract infections, including cystis fibrosis and pulmonary tuberculosis patients. $\mathrm{C} 3 \mathrm{~A}$ and $\mathrm{C} 3 \mathrm{~B}$ strains were exclusively isolated from patients suffering respiratory tract infections, in France and Malaysia, respectively. Therefore, clusters specify the clinical form and geographical origin of the infection.

Altogether, genomics analyses revealed a more heterogeneous structure of $M$. abscessus complex than the one currently suggested by the nomenclature, which recognizes only two subspecies within $M$. abscessus [16]. It has been proposed that two genomes exhibiting AAI $>96 \%$ belong to the same species $[34,35]$. Therefore, AAI analysis indicates that M. abscessus is in fact comprising of three genomospecies, corresponding to previous nomenclature of $M$. abscessus (C1), M. massiliense (C2) and M. bolletii (C3). Using an AAI $<97 \%$ threshold would further determine two subspecies in M. massiliense (C2A and $\mathrm{C} 2 \mathrm{~B}$ ) and in M. bolletii (C3A and $\mathrm{C} 3 \mathrm{~B}$ ). Recent whole genome sequencing analyses of clinical isolates in Great Britain also clearly distinguished three clusters in agreement with the three here reported [8]. All these data support revaluating the taxonomy of $M$. abscessus complex, to recognize three genomospecies $M$. abscessus (C1), M. bolletii (C2), and $M$. massiliense (C3); and four unnamed subspecies C2A, C2B; C3A, C3B. 


\section{M. abscessus prophagome}

M. abscessus median GC\% content is $64.2 \%$, ranging from $62.7 \%$ (M. abscessus ATCC 19977) to 64.2\% (strain Go 06$)$. The GC\% is not characteristic of the clusters as the median $\mathrm{GC} \%$ content of $\mathrm{C} 1, \mathrm{C} 2 \mathrm{~A}$ and $\mathrm{C} 3$ is $64.2 \%$, close to the median $64.1 \% \mathrm{GC} \%$ content in C2B.

However, there is a significant $14.7 \%$ variation in the genome length from 4.8-Mb (M. abscessus M154) to 5.51-Mb (M. abscessus M24) with a median of 5.07-Mb. The median of genome size is $5.07-\mathrm{Mb}$ in $\mathrm{C} 1,4.89-\mathrm{Mb}$ in $\mathrm{C} 2 \mathrm{~A}, 5.01-$ $\mathrm{Mb}$ in $\mathrm{C} 2 \mathrm{~B}$ and 5.28-Mb in $\mathrm{C} 3$. Differences in the genome size correlate with the number of prophage regions which are detected in 13/14 M. abscessus genomes (Figure 3): $M$. abscessus M154 (M. massiliense C2A) has the smallest genome encoding no prophage whereas M. bolletii M24 (C3) has the largest genome encoding seven prophage regions (Figure 3). Prophage regions comprise up to $5 \%$ of the genome lenght in M. abscessus M172. The number of prophage regions in other genomes is diverse, ranging from one to six regions encoding putative genes in the subsystem of phages, prophages, transposable elements and plasmids, which might contribute to species diversity [36]. The mean number of prophage regions is 2 in $\mathrm{C} 1,1.33$ in $\mathrm{C} 2 \mathrm{~A}$, 3.5 in $\mathrm{C} 2 \mathrm{~B}$ and 5 in $\mathrm{C} 3$. This observation confirms the particularity of C3: M. bolletii CIP108541 ${ }^{\mathrm{T}}$ contains a $13-\mathrm{kb}$ and a 63-kb prophage whereas M. bolletii strain M24 contains seven prophage regions including one $17-\mathrm{kb}$ region homologous to the $M$. bolletii CIP $108541^{\mathrm{T}} 13-\mathrm{kb}$ region and a $27-\mathrm{kb}$ region homologous to the $M$. massiliense CCUG 48898 50-kb region [37,38] (Table 4). M. abscessus genomes encode putative phage-related genes necessary for phage replication as well as phage-tail protein, phage endolysin, capsid proteins (major protein and scaffold proteins) and phage tape measure protein. Both ends of this region encode putative phage integrases. $M$. abscessus genomes encode small prophage-like regions. However, only $M$. bolletii has been reported to produce a mycobacteriophage that we named Araucaria after we recently resolved its electron microscopy 3D structure [39]. $M$. abscessus M94 genome harbours one particular pseudotRNA spanning the region 51,150-57,394 in contig 33, which is not observed in the other $M$. abscessus genomes [40]. Phages have been reported to increase virulence of their host and encode antimicrobial resistance genes [41]. In $M$. abscessus however, no such genes were identified but phages could be targeted for the differentiation between the three M. abscessus genomospecies.

\section{M. abscessus complex resistome}

As all mycobacteria, M. abscessus complex is embedded into a hydrophobic cell wall barrier to hydrophilic antibiotics. Accordingly, M. abscessus is multidrug resistant organisms exhibiting different drug resistance [42-44]. $M$. abscessus genomes encode many proteins potentially involved in drug-efflux systems, including members of the major facilitator family, $\mathrm{ABC}$ transporters and $\mathrm{MmpL}$ proteins; Small Multidrug Resistance-family, a family of lipophilic drug efflux proteins [45]; and a multidrug resistance stp protein similar to $M$. tuberculosis involved in spectinomycin and tetracycline resistance [46]. M. abscessus, $M$. bolletii and M. massiliense were reported to be in-vitro susceptible to amikacin; however, comparison with the $M$.

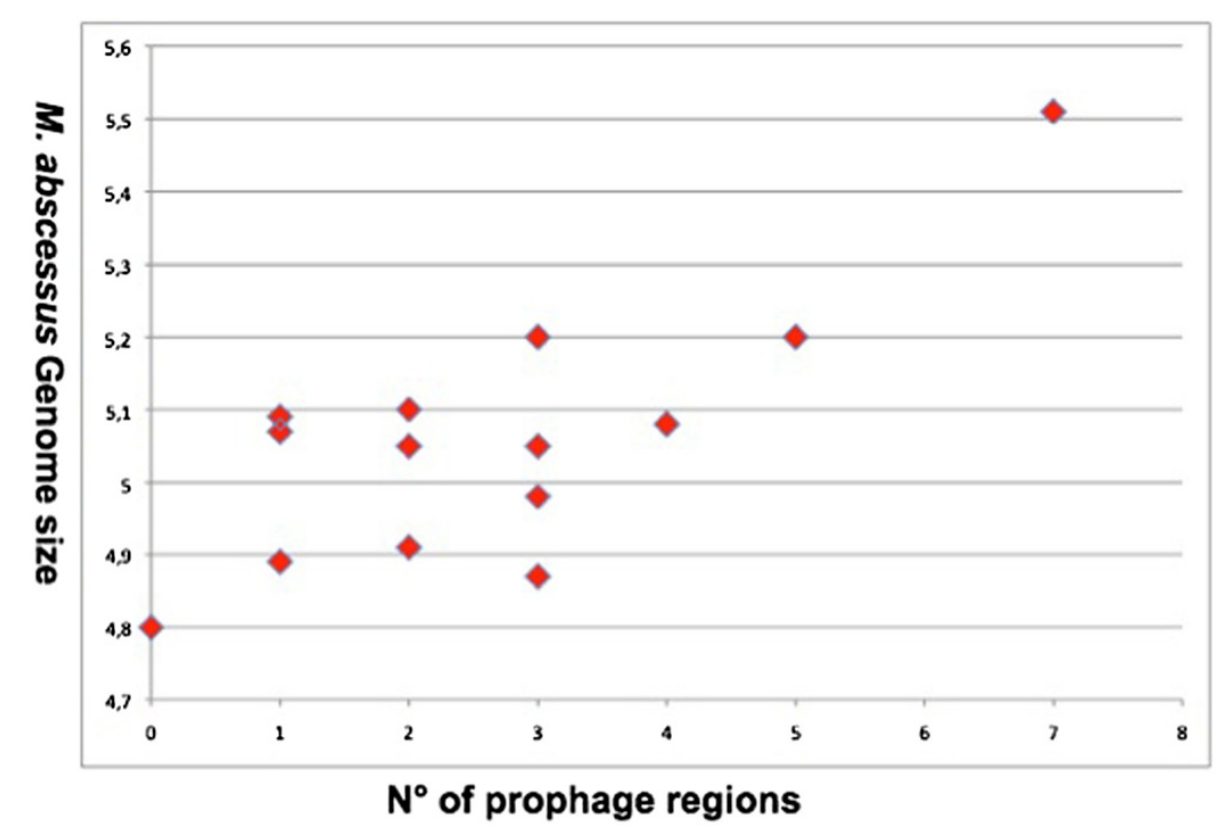

Figure 3 Correlation between Mycobacterium abscessus genomes size ( $y$ axis) and the number of prophages ( $x$ axis). 
Table 4 Mycobacterium abscessus prophages

\begin{tabular}{|c|c|c|c|c|c|c|}
\hline Clusters & & Strain & Region & Region_length & CDS & Region_position \\
\hline \multirow[t]{10}{*}{ M. abscessus } & $\mathrm{C} 1$ & M. abscessus $T$ & 1 & $81 \mathrm{~Kb}$ & 110 & 1754551-1835095 \\
\hline & & M93 & 1 & $16.4 \mathrm{~Kb}$ & 33 & $197463-213867$ \\
\hline & & & 2 & $38 \mathrm{~Kb}$ & 51 & $232006-270072$ \\
\hline & & & 3 & $53 \mathrm{~Kb}$ & 70 & $1762720-1815780$ \\
\hline & & & 4 & $20.2 \mathrm{~Kb}$ & 26 & 1820768-1841058 \\
\hline & & M94 & 1 & $58.3 \mathrm{~Kb}$ & 84 & 1039523-1097850 \\
\hline & & & 2 & $79.4 \mathrm{~Kb}$ & 99 & $4959719-5039151$ \\
\hline & & M152 & 1 & $48.9 \mathrm{~Kb}$ & 53 & $1897722-1946683$ \\
\hline & & & 2 & $34.9 \mathrm{~Kb}$ & 44 & $4784847-4819818$ \\
\hline & & Go06 & 1 & $65 \mathrm{~Kb}$ & 44 & 1768158-1833157 \\
\hline \multirow[t]{19}{*}{ M. massiliense } & $\mathrm{C} 2 \mathrm{~A}$ & M. massiliense $T$ & 1 & $12.5 \mathrm{~Kb}$ & 21 & $1600973-1613514$ \\
\hline & & & 2 & $31.3 \mathrm{~Kb}$ & 33 & $1620002-1651385$ \\
\hline & & & 3 & $50.4 \mathrm{~Kb}$ & 69 & $3907205-3957680$ \\
\hline & & M18 & 1 & $62.8 \mathrm{~Kb}$ & 67 & $4702725-4765592$ \\
\hline & & M154 & 0 & 0 & 0 & 0 \\
\hline & $\mathrm{C} 2 \mathrm{~B}$ & M115 & 1 & $11.6 \mathrm{~Kb}$ & 10 & $1416841-1428481$ \\
\hline & & & 2 & $77.1 \mathrm{~Kb}$ & 102 & $1624644-1701770$ \\
\hline & & & 3 & $55.3 \mathrm{~Kb}$ & 79 & $3356346-3411651$ \\
\hline & & M172 & 1 & $55.1 \mathrm{~Kb}$ & 74 & $502478-557677$ \\
\hline & & & 2 & $50.7 \mathrm{~Kb}$ & 50 & $546109-596832$ \\
\hline & & & 3 & $59 \mathrm{~Kb}$ & 67 & 1934186-1993225 \\
\hline & & & 4 & $31.1 \mathrm{~Kb}$ & 33 & $2050376-2081567$ \\
\hline & & & 5 & $39.4 \mathrm{~Kb}$ & 45 & $3711805-3751246$ \\
\hline & & & 6 & $19.6 \mathrm{~Kb}$ & 40 & $3753466-3773078$ \\
\hline & & M47J26 & 1 & $39.9 \mathrm{~Kb}$ & 48 & $1066714-1106668$ \\
\hline & & & 2 & $12.4 \mathrm{~Kb}$ & 16 & $3596408-3608873$ \\
\hline & & & 3 & $41.4 \mathrm{~Kb}$ & 42 & 3823414-3864899 \\
\hline & & M139 & 1 & $35.9 \mathrm{~Kb}$ & 43 & 2906235-2942215 \\
\hline & & & 2 & $12.5 \mathrm{~Kb}$ & 17 & $5033731-5046263$ \\
\hline \multirow[t]{10}{*}{ M. bolletii } & C3A & M. bolletii $T$ & 1 & $41.6 \mathrm{~Kb}$ & 47 & $1684736-1726377$ \\
\hline & & & 2 & $20.9 \mathrm{~Kb}$ & 38 & $1727918-1748849$ \\
\hline & & & 3 & $12.4 \mathrm{~Kb}$ & 16 & $3641720-3654182$ \\
\hline & C3B & M24 & 1 & $37.1 \mathrm{~Kb}$ & 51 & 560940-598047 \\
\hline & & & 2 & $37 \mathrm{~Kb}$ & 37 & $1680197-1717263$ \\
\hline & & & 3 & $17 \mathrm{~Kb}$ & 21 & $3830340-3847343$ \\
\hline & & & 4 & $18.1 \mathrm{~Kb}$ & 34 & $5051771-5069955$ \\
\hline & & & 5 & $26 \mathrm{~Kb}$ & 35 & 5155113-5181190 \\
\hline & & & 6 & $19.2 \mathrm{~Kb}$ & 26 & 5213195-5232444 \\
\hline & & & 7 & $26.5 \mathrm{~Kb}$ & 33 & 5312024-5338593 \\
\hline
\end{tabular}

tuberculosis $\mathrm{H} 37 \mathrm{Rv}$ resistome and the antibiotic resistance databases indicate that $M$. abscessus encodes an aminoglycoside 29-N-acetyltransferase and aminoglycoside phosphotransferases involved in resistance to aminoglycosides. Also, genetic analyses disclosed $16 \mathrm{~S}$ rRNA gene mutations conferring aminoglycoside resistance [4,5,47]. Indeed, the presence of a single rRNA operon in all of the $M$. abscessus genomes favours the occurrence of dominant mutations conferring resistance to aminoglycosides and macrolides. $M$. abscessus genomes encode a rifampin 
ADP-ribosyl transferase and monooxygenases potentially involved in resistance to rifampin and tetracyclines. Moreover, $M$. abscessus genomes encode three tet $(\mathrm{M})$ genes conferring resistance to tetracyclyine and doxycycline; the number of tet $(\mathrm{M})$ genes was correlated to the resistance to cyclines in Escherichia coli [48]. However, M. massiliense was reported to be susceptible and $M$. abscessus and $M$. bolletii to be resistant to doxycycline [49]. M. abscessus genomes encode resistance to fusidic acid, glycopeptides, MLS (Macrolide-Lincosamide-StreptograminB), phenicols, rifampicin, sulphonamide and trimethoprim. Also, $M$. abscessus genomes encode FolP homologs conferring resistance to cotrimoxazole, homolog of UDP-N- acetylglucosamine 1-carboxyvinyltransferase, a MurA protein conferring resistance to fosfomycin and homologs of 23S rRNA methylases conferring resistance to macrolides. Also, M. abscessus genome encodes an erm(41) gene which mutations were reported to confer clarithromycin resistance [50]. Invitro tests showed that $M$. massiliense clinical isolates could be distinguished from $M$. abscessus isolates for their susceptibility to ciprofloxacin [51] whereas $M$. bolletii isolates were reported to be resistant to all quinolones [52]. A mutation at codon 90 in gyrA gene was reported in clinical isolates of $M$. abscessus exhibiting high resistance to ciprofloxacin [51]. This observation contrasts with our genome analysis, which found no such mutations, suggesting that other mechanisms of resistance may be involved in highlevel resistance to quinolones [52]. Accordingly, we found that $M$. abscessus mycobacteria encode qepA2, a plasmidic gene conferring quinolone resistance in gram-negative bacteria [53]. M. abscessus mycobacteria were reported to be in-vitro resistant to penicillin, amoxicillin, cefoxitin, ceftriaxone, cefotaxime and imipenen $[4,5]$. This contrasts with the fact that they encode Penicillin-binding proteins (PBPs), targets for $\beta$-lactam antibiotics (except for tabtoxinine- $\beta$ lactam, which inhibits glutamine synthetase), which are essential for peptidoglycan synthesis [54,55]. M. abscessus genomes encode an Ambler class A $\beta$-lactamase homologous to $\beta$-lactamases in gram-negative bacteria and to two $\beta$-lactamases in $M$. tuberculosis. $\beta$-lactamases inhibitors have not been evaluated against M. abscessus sensu lato mycobacteria.

\section{Genome-based analysis of host-interactions}

M. abscessus are ubiquitous environmental organisms in soil and water [9] where they may have to cope with amoeba: M. chelonae, $M$. abscessus, $M$. massiliense and $M$. immunogenum were reported to survive within Acanthamoeba polyphaga tropohozoites and cysts [5]. Accordingly, our analyses indicate that M. abscessus genomes encode factors implicated in host interactions. The mean number of genes encoding proline-glutamate $(\mathrm{PE})$, proline-proline glutamate (PPE), 10-kDa lipoprotein antigen precursor $(\mathrm{LpqH})$, Mammalian Cell Entry
(MCE), oxidoreductase (Yrbe) and type VII secretion system is of 70 in C1, 80 in C2A, 74 in C2B and 93 in C3. In M. abscessus, rough colonies lack mmpL4 (a gene required for glycopeptidolipid biosynthesis) and lost surface colonization, replication into human macrophages and stimulation of innate immune response; these observations suggested that glycopeptidolipid was a virulence factor [56-58]. Accordingly, glycopeptidolipids are required for sliding motility [59] and biofilm formation [60]. Glycopeptidolipids have also been suspected to inhibit phagocytosis of $M$. avium subsp. avium [61]. M. abscessus genomes encode MCE proteins similar to $M$. tuberculosis H37Rv. MCE operon promotes internalization of $M$. tuberculosis by mammalian cells [62] and initiates rapid induction of transcription of genes involved in substrate trafficking [63]. The number of mce operons which correlated with pathogenicity [64], varies from six in C2B to 13 in C3. In parallel, M. abscessus genomes encode 12 (C1) to 21 (C3A, C3B) copies of Yrbe proteins. As for secretion systems, recent evidences showed that mycobacteria evolved specialized type VII secretion systems to transport extracellular proteins across the cell wall [65]. Type VII secretion systems ESX-1 and ESX-5 are involved in cell-tocell migration of $M$. tuberculosis $[65,66]$. In $M$. abscessus, our analyses indicate that ESX-3 and ESX-4 systems are conserved (Figure 4). However, M. abscessus M139 (C2B) lacks two proteins of the ESX-3 system and $M$. abscessus M93 (C1) lacks ESAT-6 like and CFP-10-like proteins secreted by the ESX-4 system. Interestingly, M. abscessus M18 (C2A) encodes ESAT-6 and CFP-10 proteins secreted by ESX-1 system. In addition, there are two or three PE and six (M. massiliense, M. abscessus M115 or M. abscessus 47 J26) to 12 (M. bolletii M24) PPE proteins, which are reported to be involved in the virulence of $M$. tuberculosis [67]. Our analyses further indicated that proteins related to phenazine biosynthesis, homogentisate catabolism, phenylacetic acid degradation and DNA degradation might have been transferred from Actinobacteria (e.g. Rhodococcus spp., Streptomyces spp.) and pseudomonas (Pseudomonas aeruginosa and Burkholderia cepacia). Although distantly related, these bacteria share the same ecosystem as M. abscessus within cystic fibrosis microbiota.

\section{Conclusions}

Our in-depth genomic analyses indicate that M. abscessus has a non-conservative genome, suggesting the possibility of on-going transfer of additional genetic material. Unsurprisingly, $M$. abscessus has already acquired antibiotic resistance. Also, phages have mediated diversity and horizontal gene transfer which drived the rapid evolution of this complex. Indeed, gene transfers have driven the evolution of $M$. abscessus towards three different genomospecies $M$. abscessus, $M$. massiliense and M. bolletii; and the evolution of four different yet unnamed subspecies. Each 


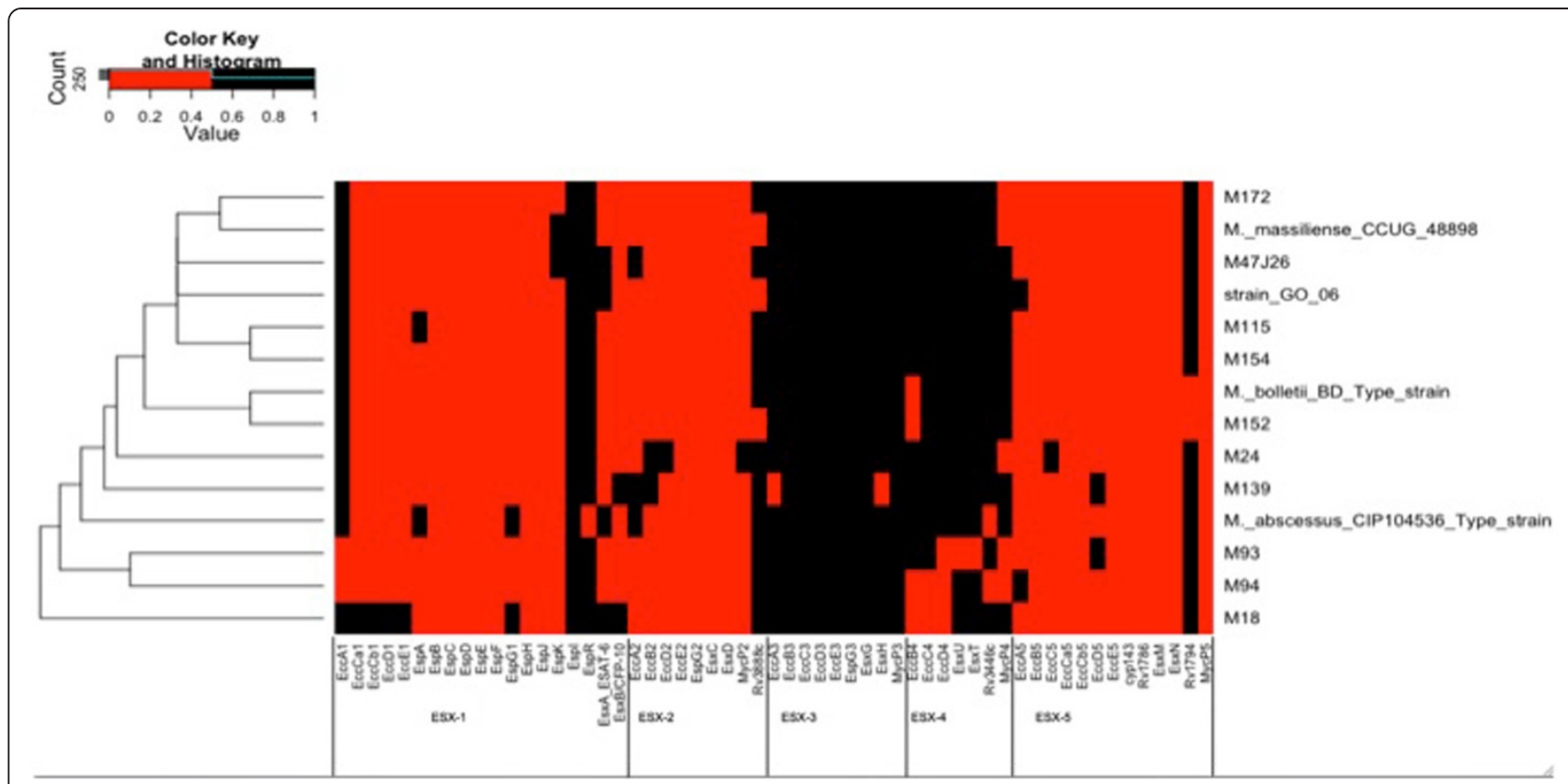

Figure 4 Heatmap clusterisation of Mycobacterium abscessus type VII secretion system compared to Mycobacterium tuberculosis H37Rv. M. abscessus strains are listed on the left side of the map.

genomospecies has its own specificities in terms of genome size, prophagome and genome content. We identified 66 genes uniquely present in each genomospecies; these genes could be used in refined detection and identification of $M$. abscessus organisms. These genomic differences support differences in host interactions and the clinical presentation of infection with $M$. massiliense (C2A and $\mathrm{C} 2 \mathrm{~B}$ ) being more virulent than the two other genomospecies. Host-interaction factors may contribute to the ability of $M$. abscessus to colonize mammalian hosts where its respiratory tract habitat put it in close proximity to other serious opportunist pathogens which can act as donors of additional host-interaction factors.

Here reported informations regarding differences between $M$. abscessus genomespecies will help understanding their pathogenesis factors and could reveal new, more specific targets for drug design and diagnosis tools.

\section{Methods}

\section{Genome dataset}

The whole genomes of $14 M$. abscessus strains were downloaded from Genbank (Table 1). The genomic sequence, either contigs or finished genomes were concatenated to one pseudogenome per genome.

\section{Prophage detection and genome annotation}

Protein sequences were predicted using prodigal software [26] to generate normalized files containing the combined protein sequences of all 14 genomes. Prophage regions were detected using PHAST software (Table 4). Predicted proteins were annotated using BLASTp against the National Center for Biotechnology Information (NCBI) nonredundant (NR) database, UNIPROT (http://www.uniprot. org/), the Clusters of Orthologous Groups (COG) [33] and a home-made antibiotic resistance gene database.

\section{Genome clustering and calculation of core genomes}

Proteome sequences were compared using by BlastP and pairwise alignments using ClustalW and the ANI was determined by the mean percentage of nucleotide sequence identity of core proteins [29]. We clustered the M. abscessus homologous genes using orthoMCL [28] on the translated protein sequences of all predicted genes with a conservative parameter value of $50 \%$ sequence identity. The determination of the different unique core genomes was based on the homology clusters found by orthoMCL.

\section{Phylogenetic analysis}

M. abscessus proteomes were aligned using Mauve software [30] to infer phylogeny using the Neighbor-Net algorithm in the package SplitsTree4 [31]. The orthologous group data found by orthoMCL were used to construct a whole-genome phylogenetic tree based on gene content. We generated a matrix of binary discrete characters (" 0 " and "1" for absence and presence, respectively) [68]. Using this matrix, we constructed a phylogenetic tree implementing the neighbor-joining (NJ) method within SplitsTree4 [31]. 


\section{Availability of supporting data}

The data set of Figure 1C supporting the results of this article is available in the TreeBase (http://treebase.org/treebase-web/home.html) repository, under the accession URL http://purl.org/phylo/treebase/phylows/study/TB2:S15632.

Reviewer access URL: http://purl.org/phylo/treebase/phylows/study/TB2:S15632? -access-code=6fa2ebc53b96e3ae4 12a8df19187ab41\&format=html.

The data sets of Figure 1A and B supporting the results of this article are included as the Additional file 1.

The data sets of Figure 4 supporting the results of this article are included as the Additional file 2.

\section{Additional files}

\section{Additional file 1: A- Aligned M. abscessus genome matrix} constructed using Mauve software to infer phylogeny using the Neighbor-Net algorithm in the package SplitsTree4. B- The matrix of binary discrete characters (" 0 " and " 1 " for absence and presence, respectively) constructed using the orthologous group data found by orthoMCL to infer phylogeny using the Neighbor-Net algorithm in the package SplitsTree4.

Additional file 2: The matrix used for Heatmap clusterisation of Mycobacterium abscessus type VII secretion system compared to Mycobacterium tuberculosis H37Rv.

\section{Competing interests}

The authors declare that they have no competing interests.

\section{Authors' contributions}

MS carried out the molecular genetic studies, participated in the sequence alignment and drafted the manuscript. MD conceived of the study, and participated in its design and coordination and drafted the manuscript. Both authors read and approved the final manuscript

\section{Acknowledgments}

MS is financially supported by the Infectiopôle Sud Foundation.

Received: 5 September 2013 Accepted: 30 April 2014

Published: 12 May 2014

\section{References}

1. Kubica GP, Baess I, Gordon RE, Jenkins PA, Kwapinski JB, McDurmont C Pattyn SR, Saito H, Silcox V, Stanford JL, Takeya K, Tsukamura M: A co-operative numerical analysis of rapidly growing mycobacteria. J Gen Microbio/ 1972, 73:55-70.

2. Ross AJ: Mycobacterium salmoniphilum sp. nov. from salmonid fishes. Am Rev Respir Dis 1960, 81:241-250.

3. Wilson RW, Steingrube VA, Böttger EC, Springer B, Brown-Elliott BA, Vincent V, Jost KC Jr, Zhang Y, Garcia MJ, Chiu SH, Onyi GO, Rossmoore H, Nash DR Wallace RJ Jr: Mycobacterium immunogenum sp. nov., a novel species related to Mycobacterium abscessus and associated with clinical disease, pseudo-outbreaks, and contaminated metalworking fluids: an international cooperative study on mycobacterial taxonomy. Int J Syst Evol Microbiol 2001, 51:1751-1764.

4. Adékambi T, Reynaud-Gaubert M, Greub G, Gevaudan MJ, La Scola B, Raoult D, Drancourt M: Amoebal coculture of "Mycobacterium massiliense" sp. nov. from the sputum of a patient with hemoptoic pneumonia. J Clin Microbiol 2004, 42:5493-5501.

5. Adékambi T, Berger $P$, Raoult $D$, Drancourt $M: r p o B$ gene sequence-based characterization of emerging non-tuberculous mycobacteria with descriptions of Mycobacterium bolletii sp. nov., Mycobacterium phocaicum sp. nov. and Mycobacterium aubagnense sp. nov. Int J Syst Evol Microbiol 2006, 56:133-143.
6. Simmon KE, Brown-Elliott BA, Ridge PG, Durtschi JD, Mann LB, Slechta ES, Steigerwalt AG, Moser BD, Whitney AM, Brown JM, Voelkerding KV, McGowan KL, Reilly AF, Kirn TJ, Butler WR, Edelstein PH, Wallace RJ Jr, Petti CA: Mycobacterium chelonae-abscessus complex associated with sinopulmonary disease, Northeastern USA. Emerg Infect Dis 2011, 9:1692-1700.

7. Petrini B: Mycobacterium abscessus: an emerging rapid-growing potential pathogen. APMIS 2006, 5:319-328.

8. Bryant JM, Grogono DM, Greaves D, Foweraker J, Roddick I, Inns T, Reacher M Aworth CSH, Curran MD, Harris SR, Peacock SJ, Parkhill J, Floto RA: Wholegenome sequencing to identify transmission of Mycobacterium abscessus between patients with cystic fibrosis: a retrospective cohort study. The Lancet 2013, 381:1551-1560.

9. Medjahed H, Gaillard JL, Reyrat JM: Mycobacterium abscessus: a new player in the mycobacterial field. Trends Microbiol 2010, 3:117-123.

10. Jassies-van der Lee A, Houwers DJ, Meertens N, van der Zanden AG, Willemse T: Localised pyogranulomatous dermatitis due to Mycobacterium abscessus in a cat: a case report. Vet J 2009, 2:304-306.

11. Albini S, Mueller S, Bornand V, Gutzwiller ME, Burnand C, Hüssy D, Abril C, Reitt K, Korczak BM, Miserez R: Cutaneous atypical mycobacteriosis due to Mycobacterium massiliense in a cat. (Article in German). Schweiz Arch Tierheilkd 2007, 12:553-558.

12. Whitaker BR, Hadfield CA, Clayton LA, Neiffer D, Stamper MA: Mycobacterium abscessus infection in an Atlantic bottlenose dolphin (Tursiops truncatus). In Proceedings of the $36^{\text {th }}$ Annual Conference of the International Association of Aquatic Animal Medicine; 2012:49-51.

13. Wiinschmann A, Armien A, Harris NB, Brown-Elliott BA, Wallace RJ Jr, Rasmussen J, Willette M, Wolf T: Disseminated panniculitis in a bottlenose dolphin (Tursiops truncatus) due to Mycobacterium chelonae infection. J Zoo Wildl Med 2008, 39:412-440.

14. Clayton LA, Stamper MA, Whitaker BR, Hadfield CA, Simons B, Mankowski LL: Mycobacterium abscessus pneumonia in an Atlantic bottlenose dolphin (Tursiops truncatus). J Zoo Wildl Med 2012, 43:961-965.

15. Zerihun MA, Nilsen $\mathrm{H}$, Hodneland S, Colquhoun DJ: Mycobacterium salmoniphilum infection in farmed Atlantic salmon, Salmo salar L. J Fish Dis 2011, 34:769-781

16. Leao SC, Tortoli E, Euzéby JP, Garcia MJ: Proposal that Mycobacterium massiliense and Mycobacterium bolletii be united and reclassified as Mycobacterium abscessus subsp. bolletii comb. nov., designation of Mycobacterium abscessus subsp. abscessus subsp. nov. and emended description of Mycobacterium abscessus. Int J Syst Evol Microbiol 2011, 61:2311-2313

17. Adékambi T, Colson P, Drancourt M: rpoB-based identification of nonpigmented and late-pigmenting rapidly growing mycobacteria. J Clin Microbiol 2003, 41:5699-5708

18. Viana-Niero C, Lima KV, Lopes ML, Rabello MC, Marsola LR, Brilhante VC, Durham AM, Leao SC: Molecular characterization of Mycobacterium massiliense and Mycobacterium bolletii in isolates collected from outbreaks of infections after laparoscopic surgeries and cosmetic procedures. J Clin Microbiol 2008, 46:850-855.

19. Arnold C, Barrett A, Cross L, Magee JG: The use of $r p o B$ sequence analysis in the differentiation of Mycobacterium abscessus and Mycobacterium chelonae: a critical judgement in cystic fibrosis? Clin Microbiol Infect 2012, 18:E131-133.

20. Leao SC, Tortoli E, Viana-Niero C, Ueki SY, Lima KV, Lopes ML, Yubero J, Menendez MC, Garcia MJ: Characterization of mycobacteria from a major Brazilian outbreak suggests that revision of the taxonomic status of members of the Mycobacterium chelonae - M. abscessus group is needed. J Clin Microbiol 2009, 47:2691-2698.

21. Macheras E, Roux AL, Ripoll F, Sivadon-Tardy V, Gutierrez C, Gaillard JL, Heym B: Inaccuracy of single-target sequencing for discriminating species of the Mycobacterium abscessus group. J Clin Microbiol 2009, 47:2596-2600.

22. Macheras E, Roux AL, Bastian S, Leão SC, Palaci M, Sivadon-Tardy V, Gutierrez C, Richter E, Rusch-Gerdes S, Pfyffer G, Bodmer T, Cambau E, Gaillard JL, Heym B: Multilocus sequence analysis and $r p o B$ sequencing of Mycobacterium abscessus (sensu lato) strains. J Clin Microbio/ 2011, 49:491-499.

23. Zelazny AM, Root JM, Shea YR, Colombo RE, Shamputa IC, Stock F, Conlan S, McNulty S, Brown-Elliott BA, Wallace RJ Jr, Olivier KN, Holland SM, Sampaio EP: Cohort study of molecular identification and typing of Mycobacterium abscessus, Mycobacterium massiliense, and Mycobacterium bolletii. J Clin Microbiol 2009, 47:1985-1995.

24. Sassi M, Ben Kahla I, Drancourt M: Mycobacterium abscessus multispacer sequence typing. BMC Microbiol 2013, 13:1-3. 
25. Ripoll F, Pasek S, Schenowitz C, Dossat C, Barbe V, Rottman M, Macheras E, Heym B, Herrmann JL, Daffé M, Brosch R, Risler JL, Gaillard JL: Nonmycobacterial virulence genes in the genome of the emerging pathogen Mycobacterium abscessus. PLoS One 2009, 4:e5660.

26. Hyatt D, Chen GL, Locascio PF, Land ML, Larimer FW, Hauser L: Prodigal: prokaryotic gene recognition and translation initiation site identification. BMC Bioinformatics 2010, 11:119.

27. Wozniak M, Wong L, Tiuryn J: CAMBer: an approach to support comparative analysis of multiple bacterial strains. BMC Genomics 2011, 12(2):S6.

28. Li L, Stoeckert CJ Jr, Roos DS: OrthoMCL: identification of ortholog groups for eukaryotic genomes. Genome Res 2003, 13:2178-2189.

29. Sentausa E, Fournier PE: Advantages and limitations of genomics in prokaryotic taxonomy. Clin Microbiol Infect 2013, 19:790-795.

30. Darling AE, Mau B, Perna NT: progressiveMauve: multiple genome alignment with gene gain, loss and rearrangement. PLoS One 2010, 5:e11147.

31. Bryant $D$, Moulton V: Neighbor-net: an agglomerative method for the construction of phylogenetic networks. Mol Biol Evol 2004, 21:255-265.

32. Raiol T, Ribeiro GM, Maranhão AQ, Bocca AL, Silva-Pereira I, Junqueira-Kipnis AP, Brigido Mde M, Kipnis A: Complete genome sequence of Mycobacterium massiliense. J Bacterio/ 2012, 194:5455

33. Tatusov RL, Fedorova ND, Jackson JD, Jacobs AR, Kiryutin B, Koonin EV Krylov DM, Mazumder R, Mekhedov SL, Nikolskaya AN, Rao BS, Smirnov S, Sverdlov AV, Vasudevan S, Wolf YI, Yin JJ, Natale DA: The COG database: an updated version includes eukaryotes. BMC Bioinformatics 2003, 4:41.

34. Konstantinidis KT, Ramette A, Tiedje JM: The bacterial species definition in the genomic era. Philos Trans R Soc Lond B Biol Sci 2006, 361:1929-1940.

35. Konstantinidis KT, Tiedje JM: Prokaryotic taxonomy and phylogeny in the genomic era: advancements and challenges ahead. Curr Opin Microbiol 2007, 10:504-509.

36. Canchaya C, Proux C, Fournous G, Bruttin A, Brüssow H: Prophage genomics. Microbiol Mol Biol Rev 2003, 67:238-276.

37. Choi GE, Cho YJ, Koh WJ, Chun J, Cho SN, Shin SJ: Draft genome sequence of Mycobacterium abscessus subsp. bolletii BD (T). J Bacteriol 2012, 194:2756-2757.

38. Wong YL, Choo SW, Tan JL, Ong CS, Ng KP, Ngeow YF: Draft genome sequence of Mycobacterium bolletii strain M24, a rapidly growing mycobacterium of contentious taxonomic status. J Bacterio/ 2012, 194:4475

39. Sassi M, Bebeacua C, Drancourt M, Cambillau C: The first structure of a mycobacteriophage, Araucaria. J Virol 2013, Epub ahead of print.

40. Choo SW, Wong YL, Leong ML, Heydari H, Ong CS, Ng KP, Ngeow YF: Analysis of the genome of Mycobacterium abscessus strain M94 reveals an uncommon cluster of tRNAs. J Bacteriol 2012, 194:5724.

41. Adékambi T, Drancourt M: Mycobacterium bolletii respiratory infections. Emerg Infect Dis 2009, 15:302-305.

42. Iyer A, Barbour E, Azhar E, Qadri I, Chaudhary A, Abuzenadah A, Kumosani T, Damanhouri G, Bahijri S, Al-Hejin A, Abdel Nour AM, Harakeh S: Bacteriophages in Escherichia coli antimicrobial resistance. Adv Bioscience Biotechnol 2013, 4:469-476

43. Kim HY, Kim BJ, Kook Y, Yun YJ, Shin JH, Kim BJ, Kook YH: Mycobacterium massiliense is differentiated from Mycobacterium abscessus and Mycobacterium bolletii by erythromycin ribosome methyltransferase gene (erm) and clarithromycin susceptibility patterns. Microbiol Immuno 2010, 54:347-353.

44. Koh WJ, Jeon K, Lee NY, Kim BJ, Kook YH, Lee SH, Park YK, Kim CK, Shin SJ, Huitt GA, Daley CL, Kwon OJ: Clinical significance of differentiation of Mycobacterium massiliense from Mycobacterium abscessus. Am J Respir Crit Care Med 2011, 183:405-410.

45. Paulsen IT, Skurray RA, Tam R, Saier MH Jr, Turner RJ, Weiner JH, Goldberg EB, Grinius LL: The SMR family: a novel family of multidrug efflux proteins involved with the efflux of lipophilic drugs. Mol Microbiol 1996, 19:1167-1175.

46. Ramón-García S, Martín C, De Rossi E, Aínsa JA: Contribution of the Rv2333c efflux pump (the Stp protein) from Mycobacterium tuberculosis to intrinsic antibiotic resistance in Mycobacterium bovis BCG. J Antimicrob Chemother 2007, 59:544-547.

47. Nessar R, Cambau E, Reyrat JM, Murray A, Gicquel B: Mycobacterium abscessus: a new antibiotic nightmare. J Antimicrob Chemother 2012, 67:810-818.

48. Hu GZ, Pan YS, Wu H, Hu H, Xu R, Yuan L, Liu JH, Feng JK: Prevalence of tetracycline resistance genes and identification of tet( $M)$ in clinical isolates of $E$. coli from sick ducks in China. J Med Microbiol 2013, 62:851-858.

49. Duarte RS, Lourenço MC, Fonseca Lde S, Leão SC, Amorim Ede L, Rocha IL, Coelho FS, Viana-Niero C, Gomes KM, da Silva MG, Lorena NS, Pitombo MB,
Ferreira RM, Garcia MH, de Oliveira GP, Lupi O, Vilaça BR, Serradas LR, Chebabo A, Marques EA, Teixeira LM, Dalcolmo M, Senna SG, Sampaio JL: Epidemic of postsurgical infections caused by Mycobacterium massiliense. J Clin Microbio/ 2009, 47:2149-2155.

50. Maurer FP, Rüegger V, Ritter C, Bloemberg GV, Böttger EC: Acquisition of clarithromycin resistance mutations in the 23S rRNA gene of Mycobacterium abscessus in the presence of inducible erm(41). J Antimicrob Chemother 2012, 67:2606-2611.

51. Monego F, Duarte RS, Nakatani SM, Araújo WN, Riediger IN, Brockelt S, Souza V, Cataldo Jl, Dias RC, Biondo AW: Molecular identification and typing of Mycobacterium massiliense isolated from postsurgical infections in Brazil. Braz J Infect Dis 2011, 15:436-441.

52. de Moura VC, da Silva MG, Gomes KM, Coelho FS, Sampaio JL, Mello FC, Lourenço MC, Amorim Ede L, Duarte RS: Phenotypic and molecular characterization of quinolone resistance in Mycobacterium abscessus subsp. bolletii recovered from postsurgical infections. J Med Microbiol 2012, 61:115-125.

53. Cattoir V, Poirel L, Nordmann P: Plasmid-mediated quinolone resistance pump QepA2 in an Escherichia coli isolate from France. Antimicrob Agents Chemother 2008, 52:3801-3804.

54. Basu J, Chattopadhyay R, Kundu M, Chakrabarti P: Purification and partial characterization of a penicillin-binding protein from Mycobacterium smegmatis. J Bacteriol 1992, 174:4829-4832.

55. Cayrou C, Henrissat B, Gouret P, Pontarotti P, Drancourt M: Peptidoglycan: a post-genomic analysis. BMC Microbiol 2012, 18:294.

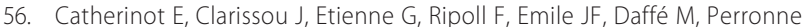
C, Soudais C, Gaillard JL, Rottman M: Hypervirulence of a rough variant of the Mycobacterium abscessus type strain. Infect Immun 2007, 75:1055-1058.

57. Nessar R, Reyrat JM, Davidson LB, Byrd TF: Deletion of the mmpL4b gene in the Mycobacterium abscessus glycopeptidolipid biosynthetic pathway results in loss of surface colonization capability, but enhanced ability to replicate in human macrophages and stimulate their innate immune response. Microbiology 2011, 157:1187-1195

58. Davidson LB, Nessar R, Kempaiah P, Perkins DJ, Byrd TF: Mycobacterium abscessus glycopeptidolipid prevents respiratory epithelial TLR2 signaling as measured by H $\beta D 2$ gene expression and IL-8 release. PLoS One 2011, 6:e29148.

59. Recht J, Martínez A, Torello S, Kolter R: Genetic analysis of sliding motility in Mycobacterium smegmatis. J Bacteriol 2000, 182:4348-4351.

60. Recht J, Kolter R: Glycopeptidolipid acetylation affects sliding motility and biofilm formation in Mycobacterium smegmatis. J Bacterio/ 2001, 183:5718-5724.

61. Villeneuve C, Etienne G, Abadie V, Montrozier H, Bordier C, Laval F, Daffe M, Maridonneau-Parini I, Astarie-Dequeker C: Surface-exposed glycopeptidolipids of Mycobacterium smegmatis specifically inhibit the phagocytosis of mycobacteria by human macrophages. Identification of a novel family of glycopeptidolipids. J Biol Chem 2003, 278:5129-5130.

62. El-Shazly S, Ahmad S, Mustafa AS, Al-Attiyah R, Krajci D: Internalization by HeLa cells of latex beads coated with mammalian cell entry (Mce) proteins encoded by the mce 3 operon of Mycobacterium tuberculosis. J Med Microbiol 2007, 56:1145-1151.

63. Stavrum R, Valvatne H, Stavrum AK, Riley LW, Ulvestad E, Jonassen I, Doherty TM Grewal HM: Mycobacterium tuberculosis Mce1 protein complex initiates rapid induction of transcription of genes involved in substrate trafficking. Genes Immun 2012, 13:496-502.

64. Ishikawa J, Yamashita A, Mikami Y, Hoshino Y, Kurita H, Hotta K, Shiba T, Hattori M: The complete genomic sequence of Nocardia farcinica IFM 10152. Proc Natl Acad Sci U S A 2004, 101:14925-14930.

65. Abdallah AM, van Pittius Gey NC, Champion PA, Cox J, Luirink J, Vandenbroucke-Grauls CM, Appelmelk BJ, Bitter W: Type VII secretionmycobacteria show the way. Nat Rev Microbiol 2007, 5:88-91.

66. Simeone R, Bottai D, Brosch R: ESX/type VII secretion systems and their role in host-pathogen interaction. Curr Opin Microbiol 2009, 12:4-10.

67. Mukhopadhyay S, Balaji KN: The PE and PPE proteins of Mycobacterium tuberculosis. Tuberculosis (Edinb) 2011, 91:441-447.

68. Snel B, Bork P, Huynen MA: Genome phylogeny based on gene content. Nat Genet 1999, 21:108-110.

doi:10.1186/1471-2164-15-359

Cite this article as: Sassi and Drancourt: Genome analysis reveals three genomospecies in Mycobacterium abscessus. BMC Genomics 2014 15:359. 